\title{
WAVE PROPAGATION ANALYSIS IN A PRESSURE-WAVE-REFRIGERATOR
}

\author{
W. ZHAO ${ }^{*}$, Z. L. JIANG, H. R. YU \\ LHD, Institute of Mechanics, Chinese Academy of Sciences, Beijing 100080, China \\ *zw@imech.ac.cn \\ T. SAITO and K. TAKAYAMA \\ SWRC, Institute of Fluid Science, Tohoku University, Sendai, 980-8577, Japan
}

Received 1 June 2005

\begin{abstract}
Pressure wave refrigerators (PWR) refrigerate the gas through periodical expansion waves. Due to its simple structure and robustness, PWR may have many potential applications if the efficiency becomes competitive with existing alternative devices. In order to improve the efficiency, the characteristics of wave propagation in a PWR are studied by experiment, numerical simulation and theoretical analysis. Based on the experimental results and numerical simulation, a simplified model is suggested, which includes the assumptions of flux-equilibrium and conservation of the free energy. This allows the independent analysis of the operation parameters and design specifics. Furthermore, the optimum operation condition can be deduced. Some considerations to improve the PWR efficiency are also given..
\end{abstract}

Keywords: Expansion wave; shock waves; refrigerator.

\section{Introduction}

The method of gas refrigeration was first tried by Power Jets Ltd in the early 1960's. The first commercial PWR was developed by two French companies (ELF Co. and Bertin Co.). A Pressure Wave Refrigerator (PWR) with rotating nozzles refrigerates gases through periodic expansion waves. It consists of a high-rate rotating distributor containing nozzles and stationary expansion tubes mounted around the distributor, see Fig.1a. High-pressure gas enters the expansion tubes when they overlap with nozzles in a process similar to the start-up of a shock tube. The strong expansion waves, generated when the exit of expansion tube is shut off by the wall of the distributor, strongly cool the inflow gas. Due to its simple structure and robustness, PWR are expected to have numerous potential applications in industry and consumer products once their efficiency becomes competitive with alternative devices. In 1980s, some experimental investigations on PWR were carried out by several researchers (Marchal 1985, Yu 1989, Fang 1991). A typically PWR description and numerical investigation was presented by Galyukov et. al. (1994, 1996). An unique PWR test facility was designed and built at the Shock Wave Research Center (SWRC) in 2003. Saito, et al (2003) studied the performance of the facility with experiments and numerical simulations, which are concentrated on evaluating the facility with varying major operation parameters. 


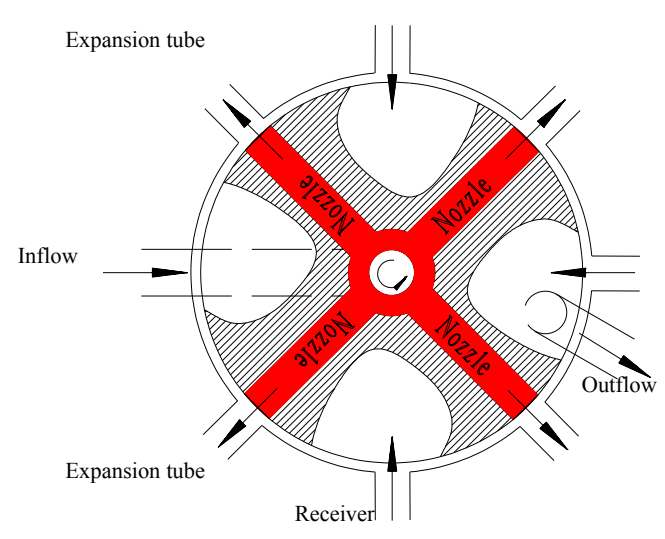

(a)

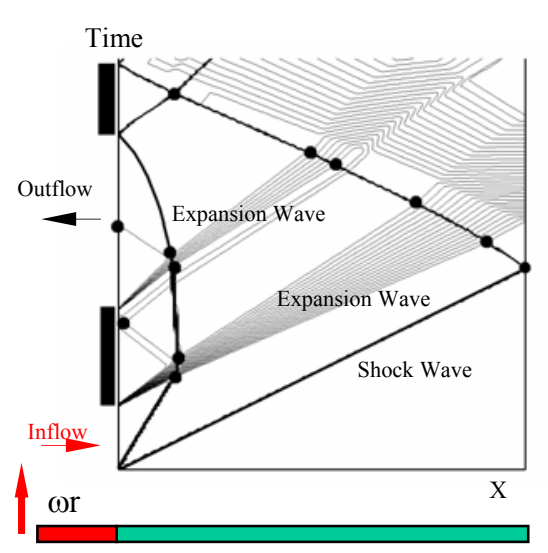

(b)

Fig. 1. (a) Design concept of a typical PWR. (b) $x$ - $t$ wave diagram in one cycle of rotating PWR operation.

The design concept of a typical PWR is shown on Fig. 1a. In order to illustrate the basic operation principles of a classical PWR, $x-t$ wave diagram in one operation cycle given by a 1-D numerical computation is shown in Fig. 1b. Transient processes caused by progressive overlapping of the gas distributor nozzles with expansion tubes have been neglected in the computation A strong expansion waves are generated when the exit of the expansion tube is shut off, the air is refrigerated by the waves. According to the results and shock tube theory, some considerations were applied in the rebuilt PWR. Meanwhile, a simplified model is proposed based on 2-D and 1-D numerical simulations.

\section{PWR Facility at SWRC}

\subsection{Experimental arrangement}

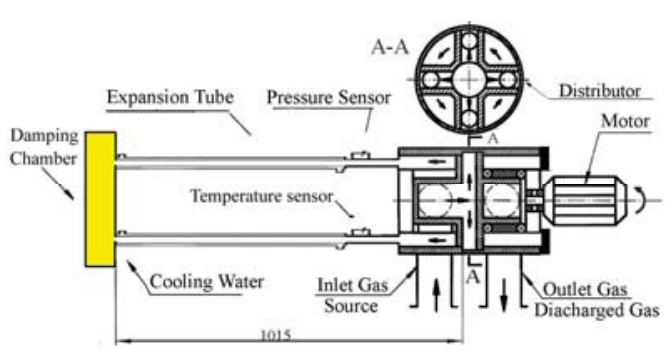

(a)

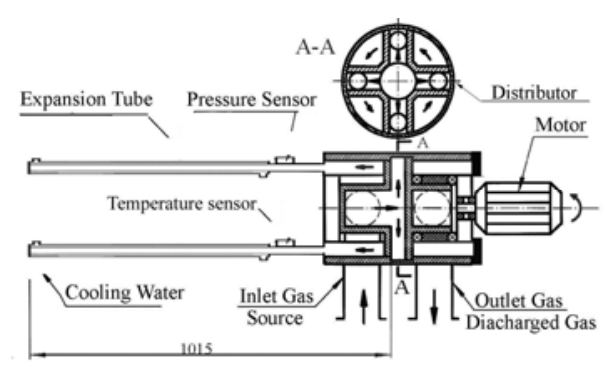

(b)

Fig. 2. Schematic of the rebuilt rotating PWR, (a) with a damping chamber, (b) without chamber.

The schematic of the rebuilt PWR is shown on Fig. 2. The total length of the expansion tube is fixed at $1.05-\mathrm{m}$ with a $0.85-\mathrm{m}$ cool-water cycle installation. The inner diameter of the expansion tube is $10-\mathrm{mm}$ while the distributor nozzle is $20-\mathrm{mm}$ in diameter. Four tubes are installed at the rotor upside. As the reflected shock wave negatively influence 
the temperature reduction, a damping chamber with $100-\mathrm{mm}$ high and 200-mm inner diameter is attached at the top of the expansion tube (downstream tube) for eliminating the reflected waves. The Diameter of the hole between the damping chamber and the expansion tube is $5-\mathrm{mm}$.

\subsection{Experimental results}

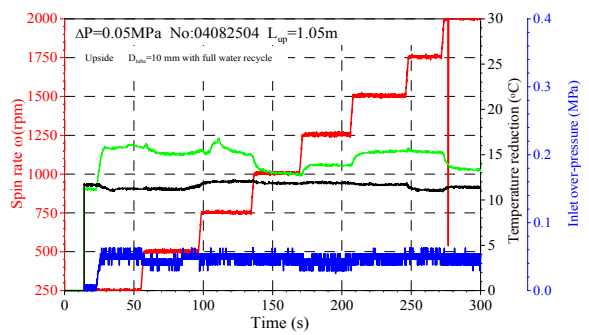

(a)

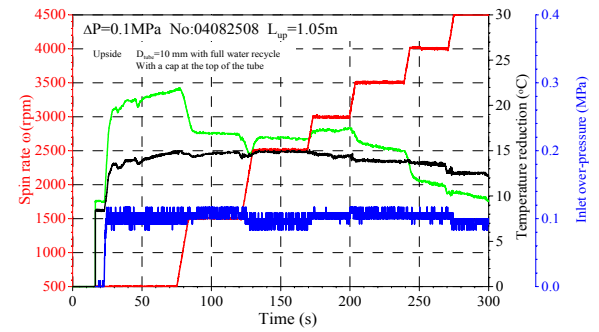

(b)

Fig. 3. Typical experimental run, (a) $\Delta \mathrm{p}=0.05 \mathrm{MPa}$, (b) $\Delta \mathrm{p}=0.10 \mathrm{MPa}$.

The typical experimental run is shown in Fig. 3. The black history corresponds to temperature reduction $\Delta \mathrm{T}_{1}$ between the inflow and outflow; and the green history is the temperature reduction $\Delta \mathrm{T}_{2}$ between the inflow inside the tube. The maximum $\Delta \mathrm{T}_{1}$ are $12^{\circ} \mathrm{C}, 15^{\circ} \mathrm{C}$ and for the $\Delta \mathrm{p}$ at $0.05 \mathrm{MPa}, 0.1 \mathrm{MPa}$ conditions, respectively. Also, the spin rate does not effect the temperature reduction $\Delta \mathrm{T}_{1}$ very obviously. Meanwhile, the optimal spin rate is decreased significantly, only within $500 \mathrm{rpm} \sim 1500 \mathrm{rpm}$.

The efficiency of the rotating PWR is increased significantly under a lower inlet pressure condition, especially, at $0.05 \mathrm{MPa}$ inlet pressure conditions and the strength of the incident shock wave (compression wave) is much stronger than that of previous results. It means the way to adopt the large ratio of the nozzle to tube is very effective.

\section{Numerical Analysis and the Simplified Model for PWR}

At first, a two-dimensional numerical analysis has been carried out The cross-sections of the nozzle and expansion tube are considered to be rectangular, and the boundary conditions at the tube exit and nozzle entry repeat cyclically in time according to the operation parameters. Typical density contours during the overlapping of the nozzle and tube are shown in Fig. 4a. A vortex can be seen to develop when the incident shock wave propagates downward.

Although many factors affect each other in a practical PWR operation, they can be analyzed independently after some reasonable simplifications:

(1) The gas in a typical PWR is an ideal gas and obeys the 1-D adiabatic flow law;

(2) The transient processes by the progressive overlapping of the nozzles with expansion tubes are neglected;

(3) The heat conduction along the tube and viscous effect are neglected, but the heat transfer between the tube end wall and the ambient is considered;

(4) No leakage in the operation; no mixing between the contact faces. 


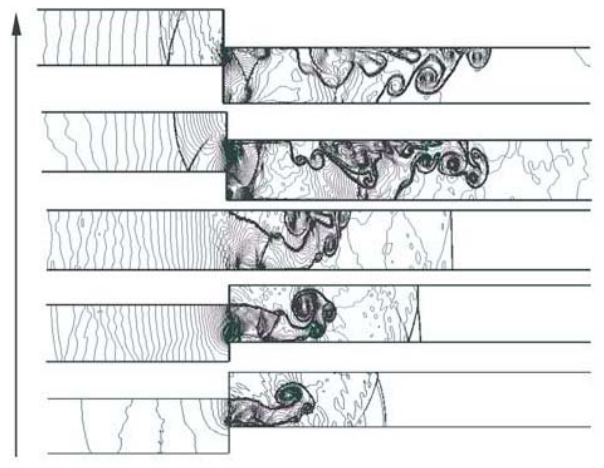

(a)

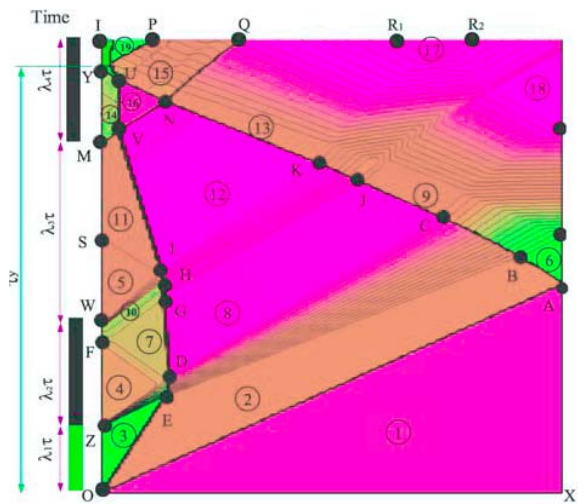

(b)

Fig. 4. (a) Waves propagation during the overlapping simulated by 2-D model. (b) Typical x-t diagram with density distribution along the expansion tube.

Based on the above simplifications and constraints, possible flow diagrams for optimal conditions are illustrated in Fig. $4 \mathrm{~b}$. We can obtain the relationships based on the shock tube theory and the mass conservation principle, which is effective and expediently to analyze the operation parameters independently.

$$
\begin{aligned}
& \alpha=\frac{T_{1}}{T_{\text {in }}^{o}} \quad \beta=\frac{P_{1}}{P_{\text {out }}} \quad \varepsilon=\frac{\lambda_{3}}{\lambda_{1}} \\
& \frac{P_{\text {in }}}{P_{\text {out }}}=\frac{1}{\beta}\left\{\frac{2 \gamma}{\gamma+1} M_{s}^{2}-\frac{\gamma-1}{\gamma+1}\right\}\left\{1-\frac{\gamma-1}{\gamma+1} \sqrt{\alpha}\left[M_{s}-\frac{1}{M_{s}}\right]\right\}^{-\frac{2 \gamma}{\gamma-1}} \\
& \int_{\tau_{O}}^{\tau_{Z}} \rho u \delta \tau+\int_{\tau_{W}}^{\tau_{M}} \rho u \delta \tau=0
\end{aligned}
$$

\section{Conclusions}

A pressure wave refrigerator has been developed at SWRC containing a rotating gasdistributor with 4 nozzles. A damping chamber is attached at the end of expansion tube. A varied cross-area is applied between the nozzle and expansion tube. The characteristic of the wave motion inside the PWR was investigated in detail with 2-D numerical simulation.

\section{References}

1. Alpher R. A. and White D. R., J. Fluid Mechanics, 8:4 (1958).

2. Galyukov A., Timofeev E., Voinovich P., Shock Waves J. 6 (1996) 301-308.

3. Li X. L., J. of Refrigeration 60 (1997) 6-12 (in Chinese).

4. Marchal P., Malek S., Viltard J. C., Oil and Gas Journal 62 (1985) 120-122.

5. Saito T., Voinovich P., Zhao W., Shibasaki K., et al., Shock Waves J. 13 (2003) 253-259. 